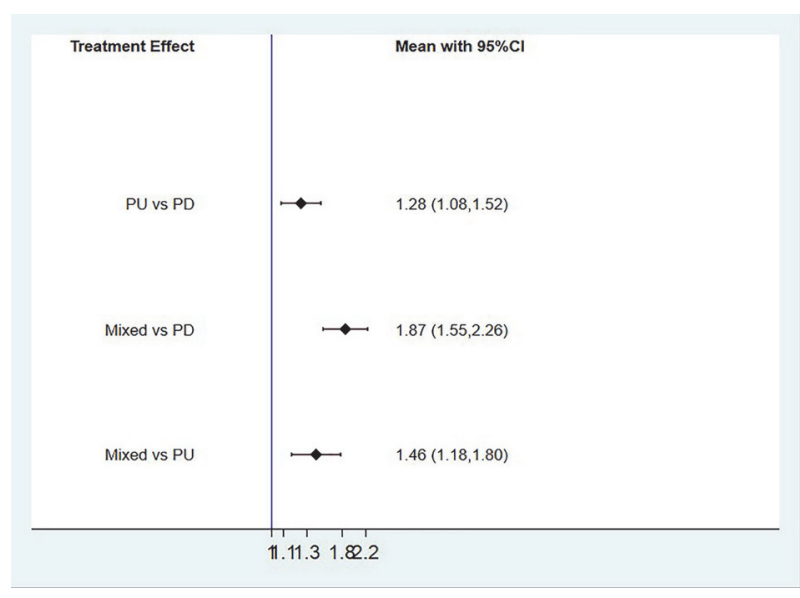

Abstract IDDF2021-ABS-0131 Figure 4

Conclusions This study identified that patients with MT EGC had an increased risk of LNM, submucosal invasion, lymphovascular invasion, and larger size compared with $\mathrm{PD}$ and $\mathrm{PU}$ EGC patients, which indicated that more attention should be focused on the clinical management of patients with MT EGC.

\section{IDDF2021-ABS-0133 POPULATIONAL DIVERSITY OF GUT MICROBIOTA IN SOUTHWEST CHINA IN THE CONTEXT OF URBANIZATION}

Yang Sun*, Yinglei Miao. First Affiliated Hospital of Kunming Medical University, China

10.1136/gutjnl-2021-IDDF.152

Background Considering the highly diverse cultural and genetic characteristics of Chinese populations, how the rapid urbanization procedure may affect the gut microbiota becomes an important question. To fill this gap, we performed a multiomic study that included over 700 adult people living in Yunnan with multiple ethnicities, to investigate the diversity of gut microbiota of these populations in the context of urbanization, and to understand how these microorganisms are connected to the health of these people.

Methods Healthy adults of Bai, Dai, Han, Hani, Miao, and Tibetan (a.k.a. Zang) were voluntarily participated in the study. The subjects were recruited from either traditional villages or the city of Kunming. Shotgun metagenomic sequencing was performed for fecal and salivary samples the participants supplied, and their dietary habits were also surveyed. A novel gene catalog was de novo assembled, and metagenomic species (MGSs) were then binned and annotated. Furthermore, the shallow depth genomic sequencing data was also recovered from the results of salivary metagenomic sequencing for metagenomic-wide association analyses.

Results Urbanization may change the taxonomic composition and functional potential of gut microbiota in a different, or even opposite, manner among different ethnicities. Further multi-omic analyses reported the significant correlations between six blood biochemical measurements and 51 intestinal MGSs, and revealed a potential connection between Catenibacterium mitsuokai, SLC52A3 gene, and prevalence of IBD.
Besides, differences in the abundance of antibiotic resistance ontologies (AROs) and carbohydrate-active enzymes of gut microbiota between lifestyles and among ethnicities were also being found.

Conclusions In this study, a novel gut microbial gene catalog of populations living in southwest China was constructed. The abundant unknown genes and MGSs in the catalog shed light on the unrecovered diversity of gut microbiota among populations. Further analyses revealed the complex connections between host genetics, ethnicities, gut microbiota and human health, and suggested the urbanization may change human gut microbiota in different ways among populations, rather in a simple, worldwide uniform manner. Moreover, the results suggested the host genetic background and lifestyle may affect the prevalence of intestinal diseases.

\section{IDDF2021-ABS-0134 ORGAN FAILURE AND INFECTED PANCREATIC NECROSIS AS DETERMINANTS OF MORTALITY IN PATIENTS WITH ACUTE PANCREATITIS: AN UPDATED SYSTEMATIC REVIEW AND META-ANALYSIS}

${ }^{1}$ Wenmo Hu*, ${ }^{1}$ Yuelun Zhang, ${ }^{1}$ Tianrui Hua, ${ }^{1}$ Guorong Chen, ${ }^{1}$ Kai Song, ${ }^{2}$ Sayali Pendharkar, ${ }^{1}$ Dong Wu. ${ }^{1}$ Peking Union Medical College Hospital, China; ${ }^{2}$ University of Auckland, New Zealand

\subsection{6/gutjnl-2021-IDDF.153}

Background In patients with acute pancreatitis, different minimally invasive approaches, and step-up approaches have been introduced and widely used to deal with infected pancreatic necrosis (IPN) in the last decade. It is unclear whether IPN has become a less important determinant of mortality relative to organ failure (OF). We aimed to statistically aggregate the evidence from published studies during the last decade to determine the relative importance of IPN and OF as determinants of mortality in patients with AP.

Methods The search for relevant studies was conducted in MEDLINE and EMBASE. The estimates are presented as relative risk (RR) or mean differences (MD) based on the type of outcomes. A two-sided $\mathrm{P}$ value less than 0.05 was regarded as statistical significance.

Results Fourteen-three studies comprising 11,601 patients with acute pancreatitis were meta-analyzed. Among them, 28\% with OF (814 of 2,901 patients) and 24\% with IPN (504 of 2,108 patients) died. Patients with OF and no IPN had a significantly higher risk of death in comparison with patients with IPN and no OF $(\mathrm{RR}=3.72 ; 95 \% \mathrm{CI}: 2.02-6.84 ; \mathrm{P}<$ $0.0001)$, though patients with OF and IPN still had a significantly higher risk of death as compared with patients with $\mathrm{OF}$ and no IPN $(\mathrm{RR}=1.50 ; 95 \% \mathrm{CI}: 1.16-1.95 ; \mathrm{P}=0.002)$ and patients with IPN and no OF $(\mathrm{RR}=4.58$; 95\% CI: 3.15-6.68; $\mathrm{P}<0.0001)$. In addition, IPN prolonged the length of stay in hospital for patients with $\mathrm{OF}(\mathrm{MD}=28.75$; 95\% CI: 2.53-54.97; $\mathrm{P}=0.032$ ).

Conclusions With the advances in the management of IPN, IPN becomes a less important determinant of mortality as compared with OF. However, IPN is still responsible for increased morbidity and prolonged hospital stay. 